\title{
AUTOMATIC CONSTRUCTION OF DIGITAL WOVEN FABRIC BY USING SEQUENTIAL YARN IMAGES
}

\author{
Zhongjian Li, Fei Yu, Ning Zhang, Yichen Lu, Ruru Pan, Weidong Gao* \\ Key Laboratory of Eco-textiles, Ministry of Education, School of Textile and Clothing, Jiangnan University, China. \\ Corresponding author: Weidong Gao, gaowd3@163.com
}

\begin{abstract}
:
In this article, a computerized method is proposed for simulating digital woven fabric (DWF) based on sequential yarn images captured from a moving yarn. A mathematical model of woven fabric structure is established by assuming that the crimped shape of yarns in weave structure is elastica, and the cross-sections of yarn in sequence image and fabric are circular and ellipse, respectively. The sequential yarn images, which are preprocessed and stitched first by image processing methods, are resized based on the mathematical model. Then a light intensity curve, which consists of radial curve model and axial curve model, is used to simulate the gray texture distribution of interlacing points in radial and axial directions. Finally, a Boole Matrix model is used to control the woven pattern. In the experiment, a slub yarn and a normal yarn samples with same count are applied to simulate gray texture fabrics. Then the gray fabrics are transformed to color fabrics based on three color maps. The fabric simulations are confined to single fabrics of plain, 2/2 matt, and 1/3 twill weaves.
\end{abstract}

\section{Keywords:}

Digital woven fabric simulation, image processing, elastica curve model, ellipse model, light intensity curve model

\section{Introduction}

Yarn quality is usually evaluated through measuring the coefficients of variation (CV) of the yarn mass. In general, this $C V$ value is seen as the main parameter to determine the quality of fabric. In fact, yarn with the same $\mathrm{CV}$ value may have different evenness in fabric [1]. This is due to the reality that the traditional method of detecting the $\mathrm{CV}$ value essentially measures the fiber content in the yarn rather than the yarn surface appearance [2], while in fact the appearance effect of woven fabric is reflected by the surface appearance of yarn. Therefore, in order to predict the woven fabric appearance in advance according to the yarn's quality, it is necessary to simulate the fabric based on the yarn's appearance. In textile factory, this prediction is carried out through test weaving. Nevertheless, the process of test weaving has disadvantages of exhausting procedure and lower efficiency [3]. And the weaving parameters cannot be modified.

Digital woven fabric (DWF), which is simulated from yarn sequence images, is used to display the yarn evenness in fabric and the quality of cloth cover. The application of DWF can replace the processing of test weaving and predict the effect of woven fabric appearance ahead, because simulating the fabric surface appearance that reflects the visual properties of yarn is important when making a final decision so as to arrive at the desired fabrics. The yarn appearance and fabric texture can also be showed clearly in the DWF. The design efficiency can be improved greatly with the DWF. It can bring huge economic benefits for the textile industry.

A number of studies have been carried out to simulate woven fabrics. These studies can be broadly classified into two categories: three-dimensional (3D) simulation and two- dimensional (2D) simulation. 3D fabric simulation is mainly used to display the geometry attribute and structure of fabric. It was usually obtained by creating 3D fabric models, such as the fiber inclination model for $3 D$ angle interlock weaves, the texture model for $3 \mathrm{D}$ electronic imaging, cross-sectional shape and centerline curve model for 3D yarn shape, B-splines model for $2 / 2$ twill woven fabric, and internal geometry model for 3D woven fabrics [4-10]. However, the abovementioned 3D fabric simulations were generally based on some mathematical models from given or measured yarn parameters by some algorithms rather than from the actual yarn images. The 3D fabric simulations placed the emphasis on the spatial structure of yarn in the fabric rather than the evenness of the actual yarn. They also did not take the illumination factors into account.

2D simulation is mainly used to display stereoscopic effect on a plane through some computer simulation methods. The yarn evenness and cloth cover situation after yarn made into fabric can be observed clearly in 2D simulation. At present, many of computer software programs and studies have been developed to simulate $2 \mathrm{D}$ weave fabrics. To mention a few, there were software programs from Fashion Studio, Nedgraphics, Pointcarré, and Wonder Weaves Systems that provided fabric simulations from yarn images [11]. Uster Zellweger, Zweigle, and Loepfe, on the other hand, provided fabric simulation software to examine probable fabric faults resulting from defective yarns to evaluate fabric quality [12]. There were also many researches exist [13-21] to simulate the 2D fabric. However, all these researches and software did not consider the actual yarn appearance. The yarn images used in the abovementioned fabric simulations were generally based on artificially constructed yarn images from given or measured yarn parameters by some mathematical algorithms rather than based on the reality yarn. In order to reflect more realistic 
effects of yarn structure in fabric appearance, Özdemir and Başer [11-12, 22] simulated woven fabrics using digital images of moving yarns. This technique would produce a more realistic simulation of fabric appearance. Ozkaya et al. [23] created a simple single jersey knitted fabric based on the measured total yarn hairiness and the hairiness distribution data from a CCD line-scan system. They gave a more realistic simulated fabric appearance. However, although the abovementioned studies simulated the fabric based on the actual yarn image, the simulation methods did not consider the illumination factors and the white color yarn, or the true structure of the yarn in a fabric.

To overcome the abovementioned shortcomings, a series of sequence yarn images, which are captured by an area-scan CCD camera continuously, are applied to simulate the DWFs automatically. The mathematical models, which consist of woven structure model, light intensity curve model and Boole Matrix model, are first constructed for single plain, 2/2 matt, and $1 / 3$ twill weaves. Then the yarn sequence images are preprocessed and stitched by image processing methods. Subsequently, the yarn core images, which are binary images without yarn hairiness, are resized based on the constructed woven structure model and the length of interlacing points. Then the resized binary images are filled with lightness texture based on the light intensity curve model. Finally, the Boole Matrix model is used to control the weave pattern to alter the up-down location of weft and warp interlacing points.

\section{Mathematical model}

\subsection{Woven Structure Model}

(i) Transforming circular yarn cross section to elliptical cross section. The yarn in sequence image was considered as the projection of a cylindrical surface on a plane perpendicular to the direction of viewing and parallel to yarn axis, and the yarn in fabric was considered as the projection of an elliptic cylinder surface [11-12]. In this article, the top view of fabric's structure, which is seen as its projection on the fabric plane, is

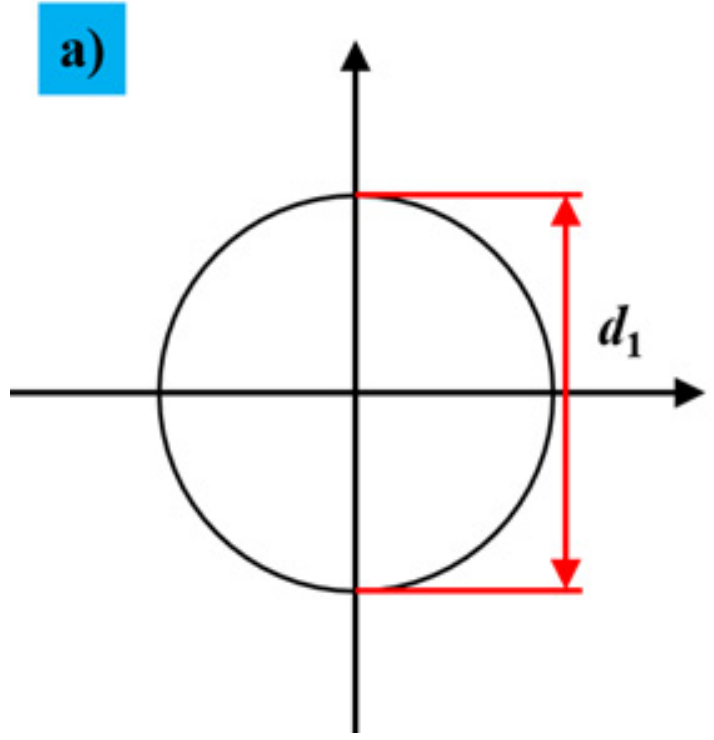

the DWF to be simulated. Therefore, the cross sections of yarn in sequence images should be transformed first to the fabric's cross sections according to the principle of circumference equal for the fabric simulation. The cross sections of yarn in sequence image and fabric, which are supposed as circular and ellipse, are shown in Figure 1 (a) and (b), respectively. In the figure, $d_{1}$ and $d_{2}$ represent the yarn diameter value in sequence image and fabric. $a$ and $b$ represent the length of two semiaxis in ellipse. Then a mathematical model developed for transforming the yarn cross section is explained in the following equations:

$$
\begin{aligned}
& C_{1}=\pi d_{1} \\
& C_{2}=2 \pi b+4(a-b) \\
& e=\frac{b}{a}
\end{aligned}
$$

where $C_{1}$ and $C_{2}$ are the circumferences of the circle and ellipse and $e$ is the eccentricity of ellipse, namely, the flattening degree of yarn in fabric, which can be measured from the actual fabric image. In this article, a value of $e=0.6$ is adopted to simulate the DWFs, which may be considered reasonable for medium sett fabrics [24]. It may, however, be changed as desired. As we know, the circumference of yarn cross section will stay the same when the actual yarn is used to weave the fabric. Thus a resizing coefficient $\left(R C_{1}\right)$ from circle to ellipse cross section can be calculated as follows:

$$
R C_{1}=\frac{d_{2}}{d_{1}}=\frac{2 a}{d_{1}}
$$

When $C_{1}=C_{2}$ and $e=0.6$, the value of $R C_{1}$ can be obtained as 1.17 .

(ii) Elastica curve model in DWF. Özdemir and Başer [1112, 22] had proved that the elastica curve model [24] were preferred being closer to reality. Thus the elastica curve model is adopted to resize the length of interlacing points in fabric. The crimped shape of the yarn in the fabric's structure is modeled by the elastica curve lying in a plane perpendicular to the fabric

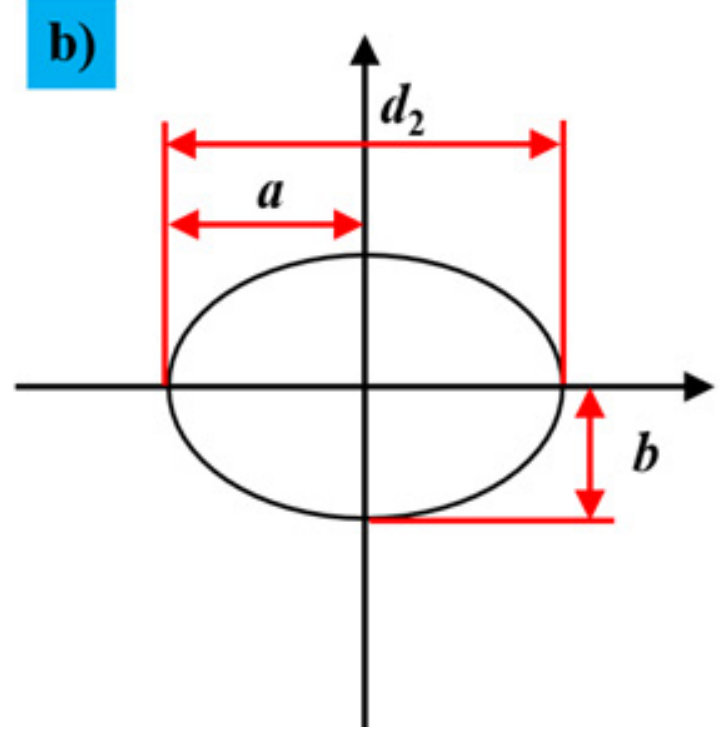

Figure 1. The cross-sections of yarn in sequence image and fabric: (a) circular; (b) ellipse. 


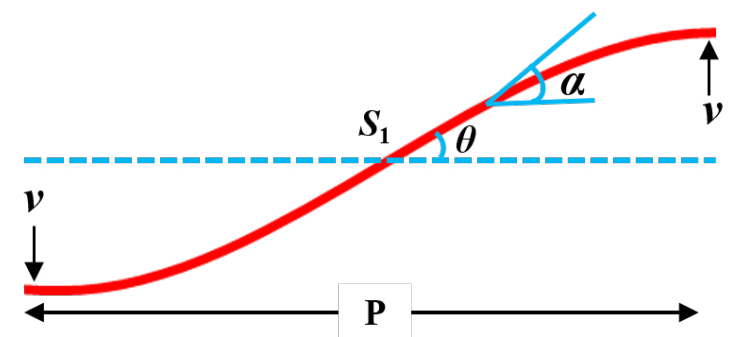

(a)
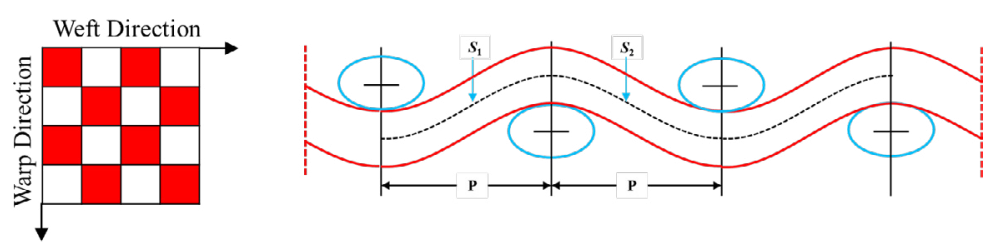

(b)
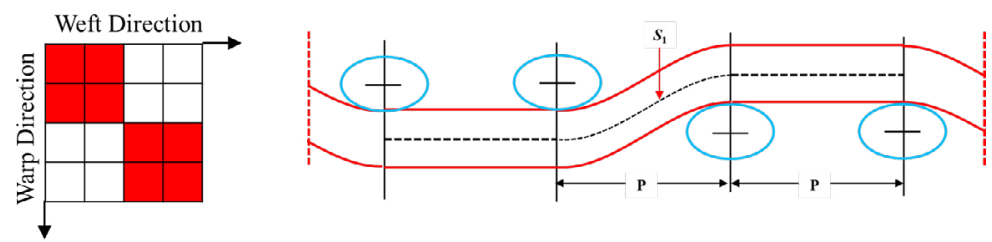

(c)
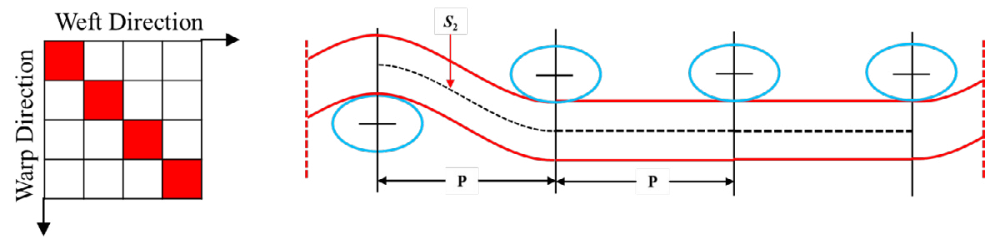

(d)

Figure 2. The elastica curve model: (a) elastica curve, (b) plain weave and the cross-sectional view of the first row of the weave, (c) $2 / 2$ matt weave and the cross-sectional view of the first row of the weave, (d) $1 / 3$ twill weave and the cross-sectional view of the first row of the weave.

surface, which is shown in Figure 2(a). The elastica curves that are expressed in the structure of plain, 2/2 matt, and 1/3 twill weaves are shown in Figure 2(b), (c), and (d), respectively.

The parametric equation of elastica curve representing the yarn in a woven fabric is given in terms of its arc length $S\left(S_{1}\right.$ or $S_{2}$ ) by the following equation ${ }^{24}$ :

$$
\frac{1}{2} R S=\int_{\alpha=0}^{\alpha=\theta} \frac{d \lambda}{\sqrt{1-k^{2} \sin ^{2} \lambda}}
$$

where

$$
\begin{aligned}
R & =\sqrt{\frac{V}{B}} \\
\lambda & =\arcsin \left[\frac{1}{k} \sin \left(\frac{\alpha}{2}+\frac{\pi}{4}\right)\right] \\
k & =\sin \left(\frac{\theta}{2}+\frac{\pi}{4}\right)
\end{aligned}
$$

Here $S$ can be $S_{1}$ or $S_{2}, V$ is the shear force at crossover point, $B$ is the flexural rigidity of the yarn, and $\theta$ is the weave angle.
Then, by substituting the values of $\alpha$ (lower limit $\alpha=0$ and upper limit $\alpha=\theta$ ) in Equation (7), we obtain

$$
\begin{aligned}
& \lambda_{0}=\arcsin \left(\frac{1}{k \sqrt{2}}\right) \\
& \frac{1}{2} R S=\int_{\lambda_{0}}^{\frac{\pi}{2}} \frac{d \lambda}{\sqrt{1-k^{2} \sin ^{2} \lambda}}
\end{aligned}
$$

The length $P$, which is the yarn spacing in fabric, in the direction of yarn axis can be expressed as [11]

$$
\frac{1}{2} R P=2 k \cos \lambda_{0}
$$

Thus the arc length $S$, which is the length of the crimped yarn between two crossover points in fabric, can be calculated as

$$
S=\frac{P \int_{\lambda_{0}}^{\frac{\pi}{2}} \frac{d \lambda}{\sqrt{1-k^{2} \sin ^{2} \lambda}}}{2 k \cos \lambda}
$$

According to Simpson's rule, suppose that the interval $[a, b]$ is split up into $n$ subintervals, with $n$ an even number. The definite integral of function $f(x)$ can be solved as [25] 

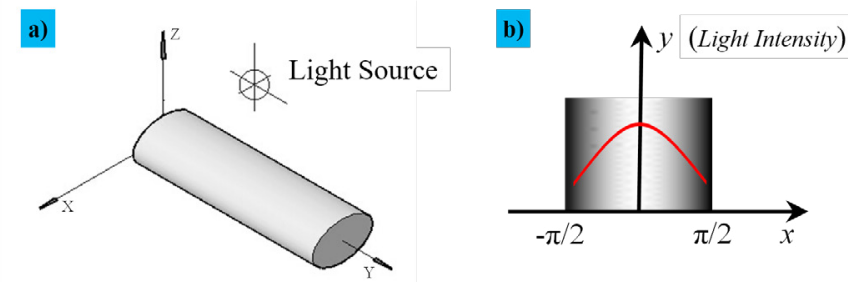

Figure 3. Elliptical cylinder model of light intensity: (a) the location of yarn and light source and (b) the light intensity distribution curve.

$\int_{a}^{b} f(x) d x \approx \frac{b-a}{3 n}\left[f\left(x_{0}\right)+4 f\left(x_{1}\right)+2 f\left(x_{2}\right)+4 f\left(x_{3}\right)+2 f\left(x_{4}\right)+\cdots+4 f\left(x_{n-1}\right)+f\left(x_{n}\right)\right]$

In this article, a weave angle of $40^{\circ}$, which represents mediumto high-set fabric [24], is adopted to simulate the DWF, and the length $P$ can be confirmed first before the simulation. Therefore, the arc length $S$, namely, the length of interlacing points in sequence yarn image, is obtained using equations (12) and (13). When simulating the DWFs, the length of interlacing point $S$ is extracted first from the sequence yarn image. And then the length $S$ is resized as $P$ to simulate the DWF by another resizing coefficient $R C_{2}$ :

$$
R C_{2}=\frac{S}{P}=\frac{\int_{\lambda_{0}}^{\frac{\pi}{2}} \frac{d \lambda}{\sqrt{1-k^{2} \sin ^{2} \lambda}}}{2 k \cos \lambda_{0}}
$$

Here, if the interlacing points are the continuous floating lines, $S=P$.

\subsection{Curve model of light intensity}

(i) Radial direction curve model. Light modeling is mainly used for the calculation of light intensity at a particular spot on a surface [26]. The light intensity distribution of interlacing point can be better displayed using a stereoscopic effect in $2 \mathrm{D}$ simulation, especially for simulating the white fabric. As the crossing yarns in fabric apply pressure to each other because of warp tension during weaving, the yarn in fabric has supposed as elliptic cylinder as shown in Figure 3(a). In the figure, a light source irradiates the yarn vertically along the direction of $z$-axis and the yarn is drawn in the direction of $y$-axis. The light intensity distribution in the appearance of the elliptical cylinder is shown in Figure 3(b) based on Lambert's cosine law [27].

According to Lambert's cosine law, the light intensity curve of any point on the radial direction of elliptical cylinder surface in the $x-y$ plane can be given by the following equation [27]:

$$
G_{1}(x)=I_{l} \times k_{d} \times \cos x \quad-\frac{\pi}{2} \leq x \leq \frac{\pi}{2}
$$

where $G_{1}(x)$ is the light intensity function, $/ /$ is the incident light intensity, $k d$ is the surface diffuse reflectance $(0 \leq k d \leq 1)$, and $x$ is an angle between the incident light direction and the surface normal.

In order to simulate the gray texture distribution in each interlacing points, the light intensity curve can be expressed as

$$
G_{2}(x)=L \sin x, \quad 0 \leq x \leq \pi
$$

Here, $L$ is the light intensity coefficient. According to Equation (16), the pixel points in interlacing points are given brightness to simulate the gray texture distribution by the following function model:

$$
W(i, j)=L_{x} \sin \left[\frac{x-B_{1}}{B_{2}-B_{1}} \times \pi\right], \quad B_{1} \leq x \leq B_{2}
$$

where $B_{1}$ and $B_{2}$ are the lower and upper boundaries when the yarn is horizontal in the sequence image. Here $L x$ is the intensity value on the centerline between $B_{1}$ and $B_{2} . W(i$, $j$ ) is the image matrix of the interlacing point, which will be simulated. $i$ is the row index; $j$ is the column index. $W(i, j)$ is the light intensity distribution matrix of weft interlacing point when $x=i$. And $W(i, j)$ is the light intensity distribution matrix of warp interlacing point when $x=j$.

(ii) Axial direction curve model for different fabrics. While the warp and weft yarn intersecting each other, the axial directions of two yarns will generate bend. In this article, the cross-sectional curves of different fabrics, which are shown in Figure 2, are used as the curve model of light intensity in axial direction. According to the cross-sectional views of the plain, $2 / 2$ matt, and 1/3 twill, which is shown in Figure 2(b), (c), and (d), respectively, the light intensity distribution of interlacing point in axial direction can be expressed as three function curves as shown in Figure 4.

The three function curves as shown in Figure 4 can be expressed as the following equations:

$y_{1}= \begin{cases}\frac{L_{s e t}}{\sqrt{1-k^{2} \sin ^{2}\left(\frac{\pi-2 \lambda_{0}}{P}(x-(i-1) \times P)+\frac{\pi}{2}+\lambda_{0}\right)}} & (i-1) \times P \leq x \leq i \times P \quad i=1,3,5 \ldots \\ \frac{L_{s e t}}{\sqrt{1-k^{2} \sin ^{2}\left(\frac{\pi-2 \lambda_{0}}{P}(x-(i-1) \times P)+\lambda_{0}\right)}} & (i-1) \times P \leq x \leq i \times P \quad i=2,4,6 \ldots\end{cases}$

$$
y_{2}=\left\{\begin{array}{cc}
\frac{L_{s e t}}{\sqrt{1-k^{2} \sin ^{2}\left(\frac{\pi-2 \lambda_{0}}{P} x+\frac{\pi}{2}+\lambda_{0}\right)}} & 0 \leq x \leq \frac{P}{2} \\
\frac{L_{1}}{\sqrt{1-k^{2} \sin ^{2}\left(\frac{\pi-2 \lambda_{0}}{P}(x-P)+\frac{\pi}{2}+\lambda_{0}\right)}} & \frac{3 P}{2} \leq x \leq 2 P \\
\frac{L_{\text {set }}}{\sqrt{1-k^{2} \sin ^{2}\left(\frac{\pi-2 \lambda_{0}}{P}(x-2 P)+\lambda_{0}\right)}} & 2 P \leq x \leq \frac{5 P}{2} \\
\frac{L_{2}}{L_{s e t}} & \frac{5 P}{2} \leq x \leq \frac{7 P}{2} \\
\sqrt{1-k^{2} \sin ^{2}\left(\frac{\pi-2 \lambda_{0}}{P}(x-3 P)+\lambda_{0}\right)} &
\end{array}\right.
$$




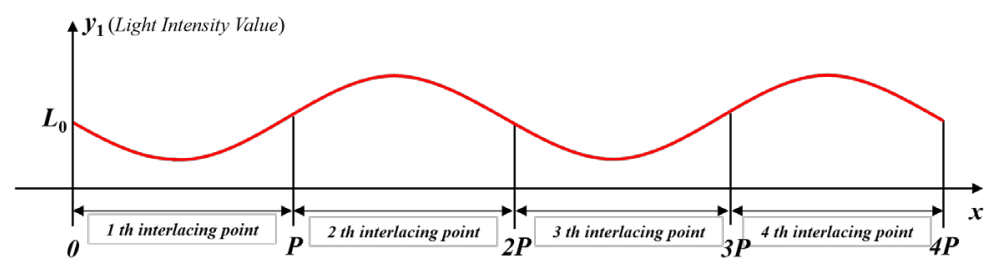

(a)

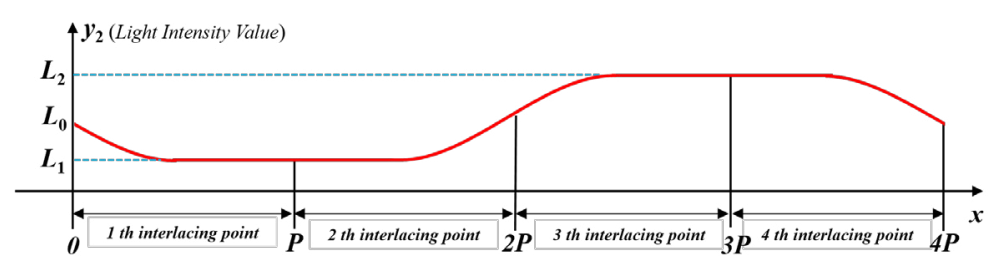

(b)

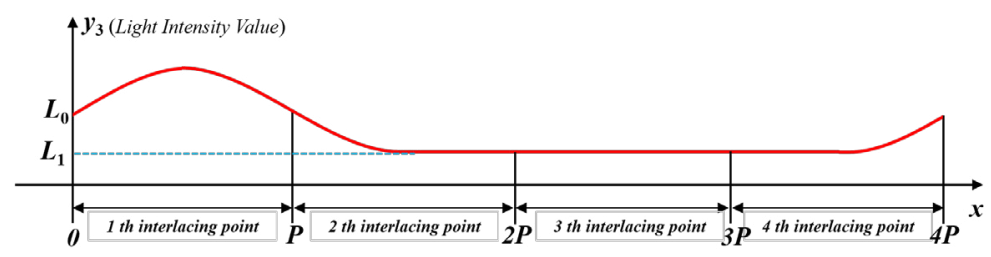

(c)

Figure 4. Three function curves of the light intensity distribution in four interlacing points: (a) plain fabric; (b) $2 / 2$ matt; and (c) $1 / 3$ twill.

$$
y_{3}= \begin{cases}\frac{L_{s e t}}{\sqrt{1-k^{2} \sin ^{2}\left(\frac{\pi-2 \lambda_{0}}{P} x+\lambda_{0}\right)}} & 0 \leq x \leq P \\ \frac{L_{s e t}}{\sqrt{1-k^{2} \sin ^{2}\left(\frac{\pi-2 \lambda_{0}}{P}(x-P)+\frac{\pi}{2}+\lambda_{0}\right)}} & P \leq x \leq \frac{3 P}{2} \\ \frac{L_{1}}{L_{s e t}} & \frac{3 P}{2} \leq x \leq \frac{7 P}{2} \leq x \leq 4 P \\ \sqrt{1-k^{2} \sin ^{2}\left(\frac{\pi-2 \lambda_{0}}{P}(x-3 P)+\lambda_{0}\right)} & \end{cases}
$$

where $i$ is the $i$ ith interlacing point, $P$ is the length of each interlacing point, and Lset is the light intensity coefficient that can be modified as desired. The calculated values of $L_{0}, L_{1}$, and $L_{2}$ are given in Equation (21):

$$
\left\{\begin{array}{l}
L_{0}=\frac{L_{s e t}}{\sqrt{1-k^{2} \sin ^{2}\left(\frac{\pi}{2}+\lambda_{0}\right)}} \\
L_{1}=L_{\text {set }} \\
L_{2}=\frac{L_{s e t}}{\sqrt{1-k^{2}}}
\end{array}\right.
$$

The three function curves can determine the intensity values of centerlines of each interlacing point in axial direction first. And then the intensity values of each interlacing point in the radial direction are calculated based on the intensity values of centerlines according to the radial direction model.

\subsection{Boole Matrix model}

A typical woven fabric weave can be expressed as a $2 D$ binary matrix [28], which is a Boole Matrix in this article. All the elements in the matrix are only 0 or 1 . "0" and " 1 " represent the weft interlacing point and the warp interlacing point, respectively.

Suppose an unit pattern matrix is $F(R w \times R j, R w, R j$ are the number of weft and warp yarns in the unit pattern, respectively), the unit Boole Matrix can be given by the following equation:

$$
F(i, j)=\left\{\begin{array}{cc}
0 & F(i, j) \text { is weft interlacing point } i=1,2, \ldots, R_{w} \\
1 & F(i, j) \text { is warp interlacing point } j=1,2, \ldots, R_{j}
\end{array}\right.
$$

For a multiple repeat pattern matrix $V\left(H_{1} \times L_{1}\right)$, the Boole Matrix can be inferred by the following equations:

$V(i, j)=\left\{\begin{array}{cc}F\left(i \backslash R_{w}, j \backslash R_{j}\right) & i \backslash R_{w} \neq 0, j \backslash R_{j} \neq 0 ; \\ F\left(i \backslash R_{w}, R_{j}\right) & i \backslash R_{w} \neq 0, j \backslash R_{j}=0 ; \\ F\left(R_{w}, j \backslash R_{j}\right) & i \backslash R_{w}=0, j \backslash R_{j} \neq 0 ;\end{array} \quad j=1,2, \ldots, H_{1}\right.$ 


$$
\begin{aligned}
& H_{1}=t_{1} \times R_{w} \\
& L_{1}=t_{2} \times R_{j}
\end{aligned}
$$

where $\lambda R_{w}$ and $\lambda R_{j}$ represent the remainders from a division of $i$ by $R_{w}$ and a division of $j$ by $R_{j}$, respectively. $H_{1} \times L_{1}$ represents the pixel size of matrix $V . t_{1}$ and $t_{2}$ are the constant and integer values.

The woven pattern can be changed by modifying the parameters of unit pattern matrix, and different multiple repeat Boole Matrices can be calculated by Equation (23). Then different patterns of DWF can be simulated more easily.

\section{Experimental details}

\subsection{Sample preparation}

Two yarn samples are captured to simulate the plain, 2/2 matt, and $1 / 3$ twill fabric weaves in the experiment. The first yarn sample (\#1) is a cotton slub yarn produced by deliberately changing the draft on the spinning frame. The yarn count is 27.8 tex, and the slub thickness is designed to be twice the yarn original diameter. The slub-length and the inter-slub separation distance are all designed to be $5 \mathrm{~mm}$. The second yarn sample (\#2) is a normal ring-spun $100 \%$ cotton yarn with a count of 27.8 tex.

\subsection{Sequence image acquisition}

An image acquisition system as shown in Figure 5 is set up to acquire continuous images of yarn in the experiment. The sequential yarn images are taken in succession along the yarn by an area-scan CCD camera with a resolution of 1,024 × 768 pixels. A closed imaging box is used as the image acquisition platform to shield the disturbance of the ambient light.

For the two experimental samples, about 20-m length of the yarns are captured by the above device. In order to facilitate the DWF simulation and obtain stitched images, the neighboring yarn images should contain a certain level (e.g., 20-50\%) of the overlapping area during acquisition. In this experiment, the frame rate of $40 \mathrm{fps}$ is set to capture the overlapping images. The exposure time is uniformly set as $200 \mu \mathrm{s}$, and the speed of the image acquisition device is uniformly set as $6 \mathrm{~m} / \mathrm{min}$. The background image and two kinds of yarn images are captured by the device, which are cropped with a suitable width to allow faster processing and are shown in Figure 6 with the size of $768 \times 600$ pixels.

\subsection{Processing yarn images for simulations}

In order to avoid the inconsistent phenomenon between two adjacent yarn images when simulating the DWF, the sequence yarn images should be stitched into one column first. Thus the

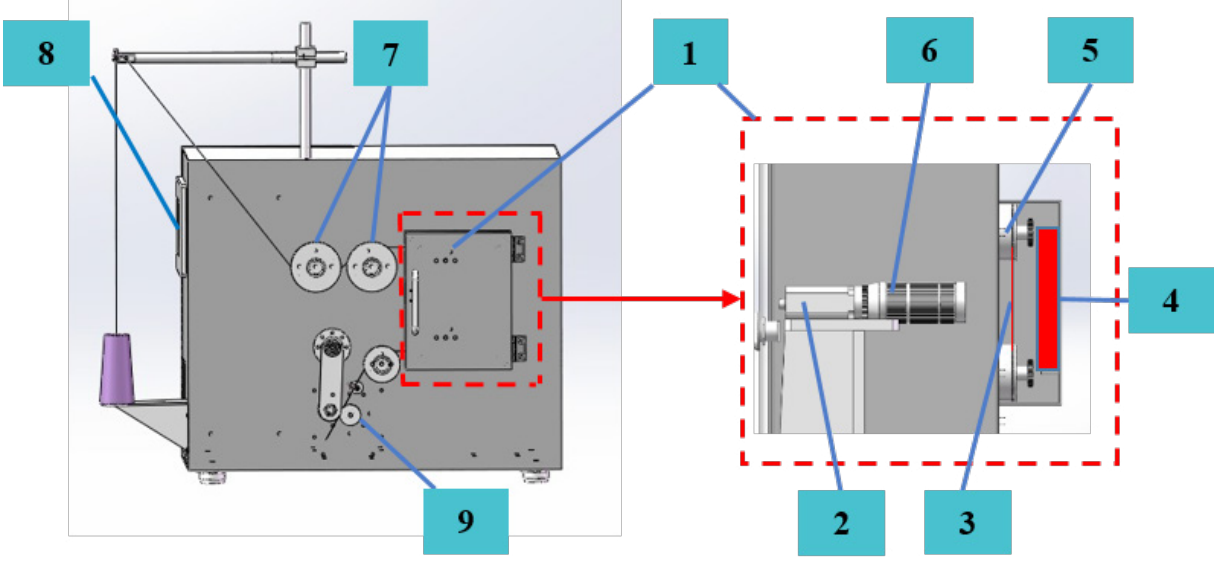

Figure 5. Yarn image acquisition device: (1) closed imaging box, (2) area-scan CCD camera sensor, (3) yarn, (4) light source, (5) yarn guiding devices, (6) camera lens, (7) yarn tension control panel, (8) touch screen, (9) output rollers with servo motor.
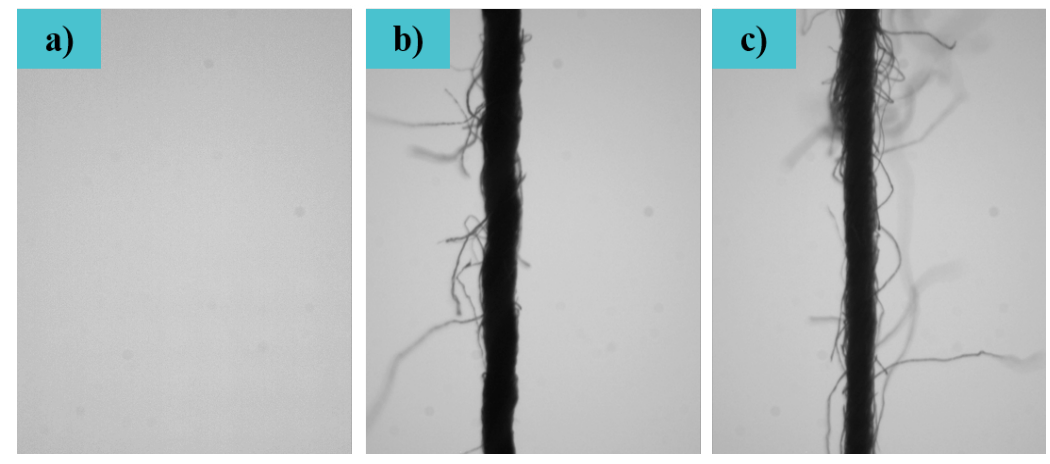

Figure 6. The captured images: (a) the background image, (b) \#1 sample, and (c) \#2 sample. 


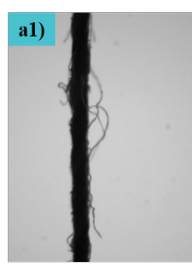

b1)
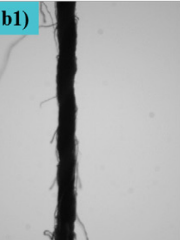

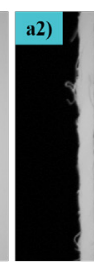

b2)

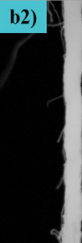

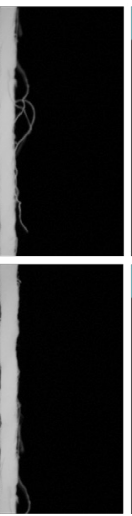
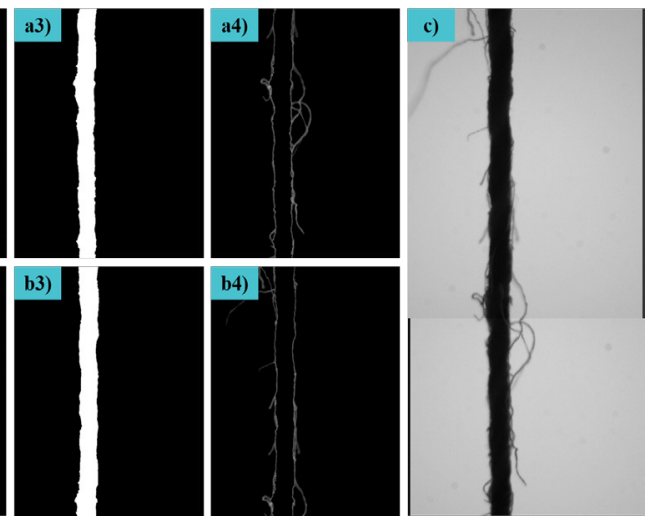

Figure 7. Image processing results of two example images: (a1), (b1) the original images; (a2), (b2) images after removing background; (a3), (b3) yarn core images; (a4), (b4) gray-hairiness images; (c) the stitched image.

image segmentation and stitching method are used to process the sequence yarn images. In our earlier work [29], the image segmentation and mosaic method had been proposed to stitch the captured sequence yarn image. Take two adjacent yarn images of sample \#1 yarn as an example (which is shown in Figure 7 (a1) and (b1)), the specific processing steps can be summarized as follows:

Step 1: Remove the background information. The background image (Figure 6(a)) is subtracted from all the original images to eliminate the impact of non-uniform brightness. The resulting images are shown in Figure 7 (a2) and (b2).

Step 2: Extract the yarn core images for simulating the DWF. The methods that consist of $K$-means clustering method and two judgment template method [30] proposed by our earlier work are applied for obtaining the yarn core images. The resulting yarn core images are shown in Figure 7 (a3) and (b3).

Step 3: Extract the gray-hairiness information for stitching the yarn sequence images. The subtraction and dot multiplication operations are executed between Figure 7 (a2)-(b2) and (a3)(b3), respectively. Then the gray-hairiness images are obtained as shown in Figure 7 (a4) and (b4).

Step 4: Stitch the adjacent yarn images based on the normalized cross-correlation (NCC) method [29, 31-33]. In this article, certain rows in the bottom of image (b4) are used as a template image to match the (a4) image. A matching coefficient matrix is calculated between the template image and the (a4) image based on the NCC method. The coordinates of the peak value in the matching coefficient matrix are found as the optimal matching points. The stitched image, which is matched by the coordinates of the peak value based on images (a4) and (b4), are shown in Figure 7(c).

After the image processing using the above steps, the optimal matching points of each image pairs (adjacent images) are obtained. These matching points are used to stitch the yarn core images for one column as the simulation information data.

\subsection{Resizing the yarn core images}

After stitching the yarn core images as one column, the yarn core images need to be resized as flatting and stereo state first.
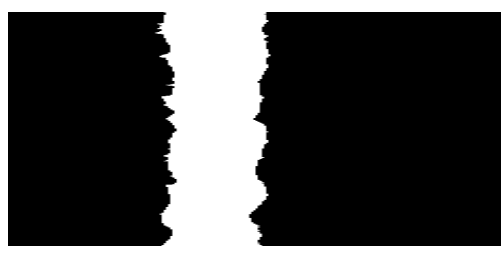

(a)
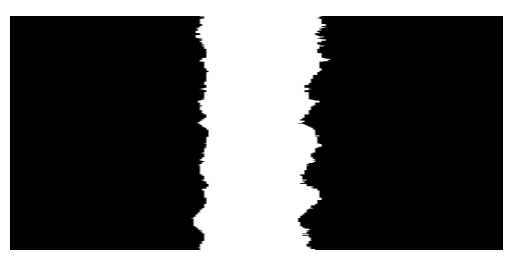

(b)
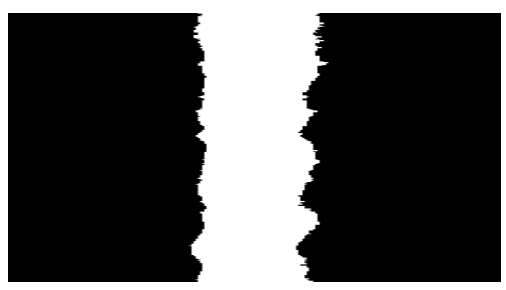

(c)
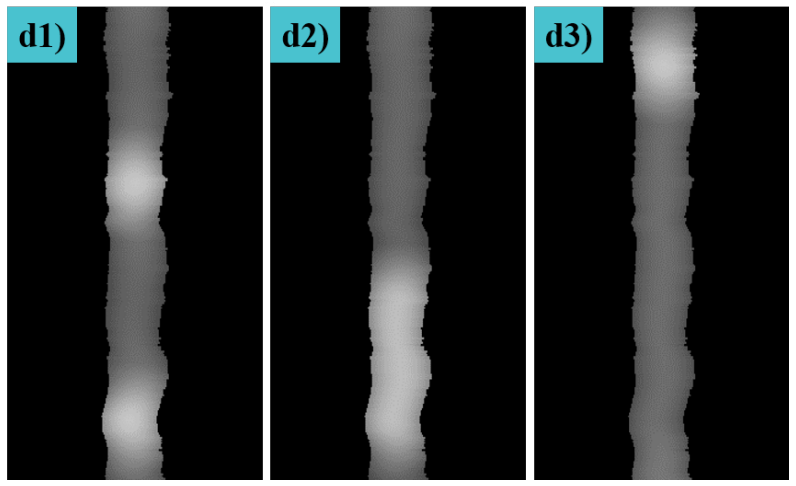

(d)

Figure 8. Image adjustment results: (a) yarn core image that includes four interlacing points, (b) image after resizing the yarn cross sections, (c) image after resizing the length, and (d) images after adding the light intensity curves: (d1) $y_{1}$ function is applied in plain weave; (d2) $y_{2}$ function is applied in $2 / 2$ matt weave; (d3) $y_{3}$ function is applied in $1 / 3$ twill weave. 
Thus the woven structure model and the light intensity curve model are substituted into the yarn core images to resize the images. The image adjustment steps are carried out as follows:

Step 1: Resize the yarn cross sections. The yarn diameter will be larger when the cross sections change from circular to ellipse. The resizing coefficient $R C_{1}\left(R C_{1}=1.17\right.$, as $\left.e=0.6\right)$ is adopted to make each diameter larger in the yarn core image. Take four interlacing points (as shown in Figure 8(a)) as an example, the resizing result of yarn cross sections is shown in Figure 8(b).

Step 2: Resize the yarn into crimp. The length of the yarn will be shorter when the yarns interweave with each other. In this article, the nearest neighbor interpolation algorithm [34] is applied to resize the yarn length. The resizing coefficient $R C_{2}$ $\left(R C_{2}=1.27\right.$, as a weave angle of $\left.\theta=40^{\circ}\right)$ is adopted to shorten the yarn length. The result of resizing of yarn length is shown in Figure 8(c).

Step 3: Add the light curve model. The radial direction and axial direction curve models are applied to Figure 8(c) based on Equations (17)-(20). The gray texture images simulated by the two curve models in four interlacing points are shown in Figure 8(d).

\subsection{Simulating the DWFs}

As all the yarn core images are resized by the above three steps, each of the interlacing point with the length of $P$ can be seen as a control point. When simulating the DWFs, the resized interlacing points are copied to a black background plane. Then the Boole Matrix model is used to determine which interlacing point is above. In the model, "0" represents a weft interlacing point that is present above a warp interlacing point and "1" represents a weft interlacing point that is present below a warp interlacing point. Examples of DWFs simulation achieved based on two yarn samples are shown in Figure 9. In the figure, the left image is constructed with $H_{1}=L_{1}=4$ in a multiple repeat pattern matrix $V$. The middle image is simulated with $H_{1}=L_{1}=$ 12 , and the right image is obtained by transforming the middle image into color image. Three color maps, called as "summer," "bone," and "copper" [34] in MATLAB 2014b, are adopted to map the gray images into color images.

\section{Discussion and Conclusions}

In this article, the plain, 2/2 matt, and 1/3 twill weaves were simulated using sequential slub yarn images and normal yarn images based on the woven structure model, light intensity curve model, and the Boole Matrix model. The sequential yarn images were captured continuously using an area-scan CCD camera first. Then these images were preprocessed and stitched by image processing methods. In this article, the yarn core images, which were binary images without yarn hairiness, were resized based on the constructed woven structure model and the length of interlacing points was set as 200 pixels. Subsequently, the resized binary image was filled with gray texture based on the light intensity curve model. Finally, the weave pattern was controlled by the Boole Matrix model, and the gray fabrics were transformed to color fabrics based on different color maps.

In the woven structure model, the cross sections of yarn in sequence image and fabric were supposed as circular and ellipse. Therefore, the yarn and fabric cross sections should be transformed first according to the principle of circumference equal for the simulation of DWFs. A resizing coefficient $\left(R C_{1}\right)$ from circle to ellipse cross section was used to resize the diameter of yarn larger. A reasonable value of the eccentricity of elliptic yarn cross section, $e=0.6$, was assumed in the application of the theory developed to calculate the resizing coefficient. Thus a value of $R C_{1}$ was calculated as 1.17 . The crimped shape of the yarn axis in the weave structure was modeled by an elastica curve lying in a plane perpendicular to the fabric surface. It had been proved that the elastica curve model were preferred being closer to reality in fabric. In the model, the length of the yarn will be shorter when the yarns interweaved with each other. A weave angle of $\theta=40^{\circ}$, which represented medium- to high-set fabrics, was adopted to calculate another resizing coefficient $R C_{2}=1.27$. The value of $R C_{2}$ was quite close to the value of 1.24 obtained from Peirce's geometry.

In the light intensity curve model, an axial direction model for different fabrics was constructed based on the three curve functions. An adjustable parameter, light intensity coefficient $L_{0}$, was set as 130 in the simulated DWFs. This axial direction model was mainly used to simulate the light intensity values of centerlines of each interlacing point in yarn axial direction. A sine curve was applied to simulate the 3D effect of the yarn in radial direction model. In Equation (17), the light intensity values of each interlacing point's centerline were used to represent the $L x$ value. These two models are very important to construct the periodically dim and brighten weft and warp interlacing points. The intensity of weft interlacing point will be brighter and the intensity of the warp interlacing point will be darker when the weft interlacing point is above the warp interlacing point, and vice versa.

The Boole Matrix model was applied to control which interlacing point is up and which is down based on different weave patterns. Finally, the gray DWFs were simulated. Then three color maps, called as "summer," "bone," and "copper" in MATLAB 2014b, were adopted to mapping the gray fabrics into color DWFs. From Figure 9, we can see that different gray values are transferred into different color values. Thus the simulated color DWFs can shade in colors. The appearance effect of yarn evenness and cloth cover situation can be clearly observed. Especially in Figure 9(a)-(c), the slub effect are displayed clearly in the DWFs.

The DWF simulation is an additional function in our yarn appearance detection system. When the yarn evenness is inspected, the yarn appearance information will be recorded. At the same time, these information data are used to simulate the DWFs. This can provide the fabric appearance situation in time. It is significantly meaningful to predict the fabric appearance from yarn appearance for the textile industry. 


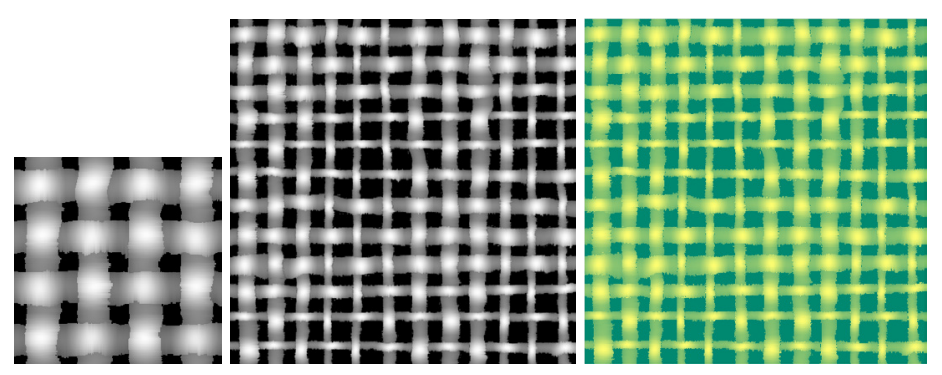

(a)

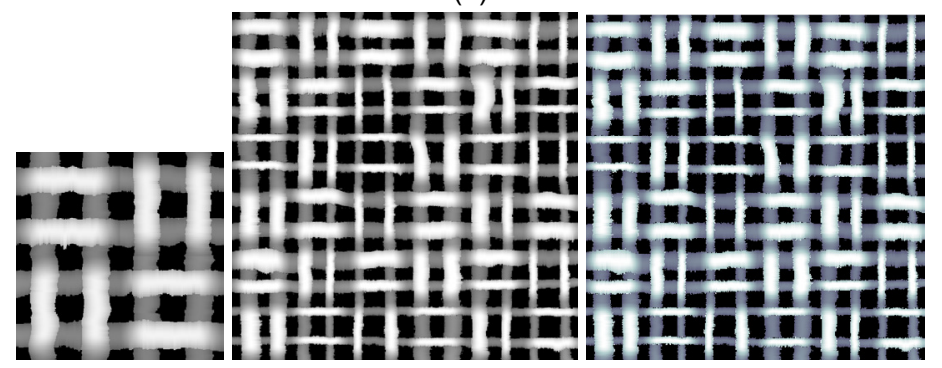

(b)

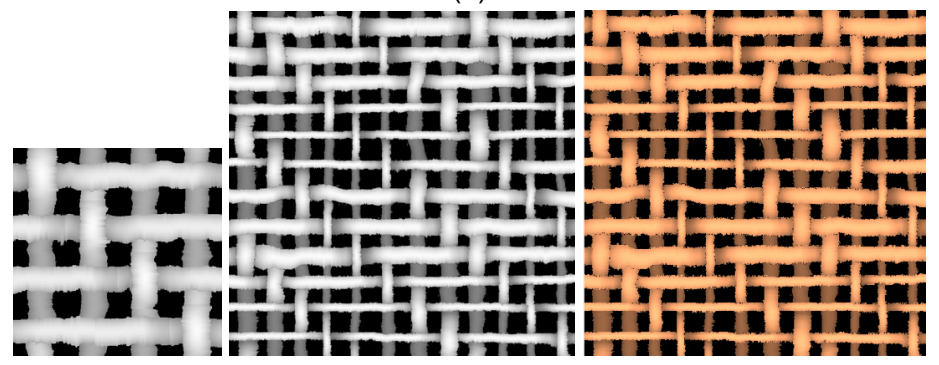

(c)

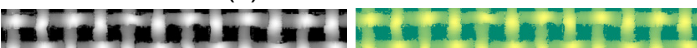

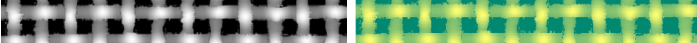

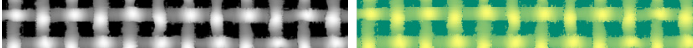

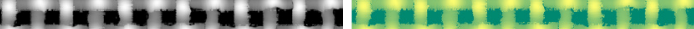

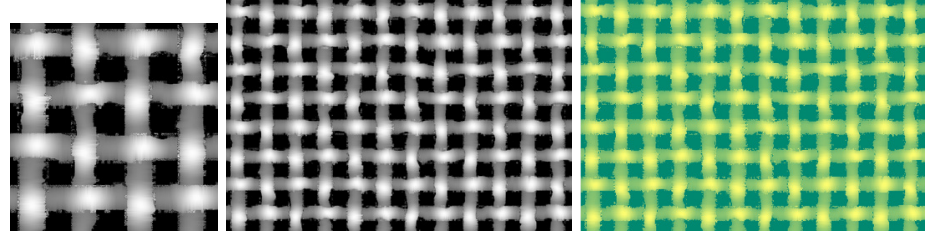

(d)

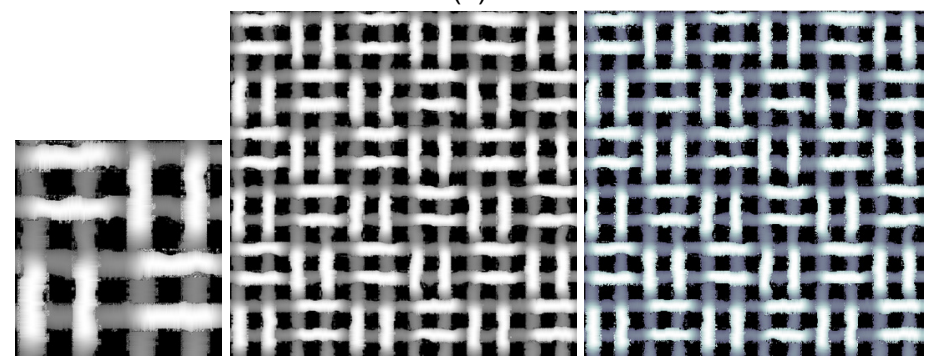

(e)

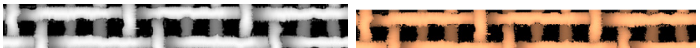

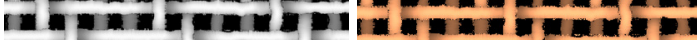

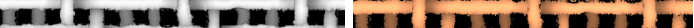

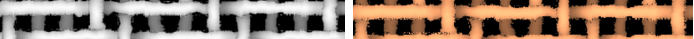

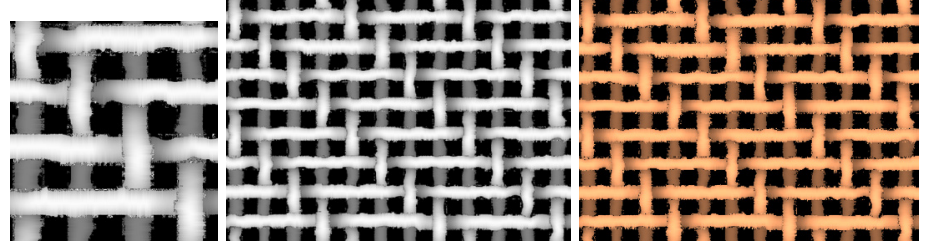

(f)

Figure 9. Examples of DWFs simulation: (a) plain weave simulated by \#1 slub yarn, (b) 2/2 matt weave simulated by \#1 slub yarn, (c) 1/3 twill weave simulated by \#1 slub yarn, (d) plain weave simulated by \#2 normal yarn, (e) $2 / 2$ matt weave simulated by \#2 normal yarn, and (f) $1 / 3$ twill weave simulated by \#2 normal yarn. 


\section{ACKNOWLEDGMENTS}

The authors would like to acknowledge the Research Innovation Program for College Graduates of Jiangsu Province (Grant No. KYLX_1132); the Postdoctoral Project (1601017A); and the Fundamental Research Funds for the Central Universities (No. JUSRP51631A).

\section{References}

[1] Liu J., Jiang H., Pan R., et al. (2012). Evaluation of yarn evenness in fabric based on image processing. Textile Research Journal, 82, 1026-1037.

[2] Carvalho V., Monteiro J.L., and Soares F.O., (2008). Yarn evenness parameters evaluation: A new approach. Textile Research Journal, 78, 119-120.

[3] Shaker K., Nawab Y., Javaid M.U., et al. (2015). Development of 3D Woven Fabric based Pressure Switch. Autex Research Journal, 15,148-152.

[4] Whitney T.J. and Chou T.W., (1989). Modeling of 3D angle interlock textile structural composites. Journal of Composite Materials, 23, 890.

[5] Suh M.W., Jasper W., and Cherkassky A., (2003). 3-D Electronic Imaging of Fabric Qualities by On-Line Yarn Data. "Annual Report", Textile Center, PA, November.

[6] Adanur S. and Liao T., (1998). 3D modeling of textile composite preforms. Composites Part B Engineering, 29, 787-793.

[7] Turan R.B. and Başer G., (2010). Three-dimensional computer simulation of 2/2 twill woven fabric by using B-splines. Journal of the Textile Institute, 101, 870-881.

[8] Dash B.P., Behera B.K., Mishra R., et al. (2013). Modeling of internal geometry of $3 D$ woven fabrics by computation method. Journal of the Textile Institute, 104, 312-321.

[9] Adanur S. and Vakalapudi J.S., (2013). Woven fabric design and analysis in $3 D$ virtual reality. Part 1: computer aided design and modeling of interlaced structures. Journal of the Textile Institute, 104, 715-723.

[10] Chen X.G., (2011). Mathematical modelling of 3D woven fabrics for CAD/CAM software. Textile Research Journal, $81,42-50$.

[11] Özdemir H. and Başer G., (2008). Computer Simulation of Woven Fabric Appearances Based on Digital Video Camera Recordings of Moving Yarns. Textile Research Journal, 78, 152-153.

[12] Özdemir H. and Başer G., (2009). Computer simulation of plain woven fabric appearance from yarn photographs. Journal of the Textile Institute, 100, 282-292.

[13] Pan R., Zhu B., Li Z., et al. (2015). A simulation method of plain fabric texture for image analysis. Industria Textilă, 66, 28-31.

[14] Jasper W., Suh, M.W., and Woo, J.L., (2000). Real Time Characterization and Data Compression Using Wavelets, "Annual Report", Textile Center, PA, November.

[15] Suh M.W. and Kim J., (1996). Fabric Image Simulation by Wavelet Analysis of Yarn Profiles, in "Proceedings of the 9th EFS System Research Forum", Raleigh, NC, November.

[16] Suh M.W. and Kim J., (1997). Creation of Virtual Fabrics by Wavelet Analysis of Spun Yarn Density Signals, in "Proceedings of the 10th EFS System Research Forum", Raleigh, NC, November.
[17] Moussa A., Dupont D., Steen D., et al. (2004). Modeling and Simulation of Woven Structure Using Fourier Transform, in "Proceedings of the World Textile Conference-4th AUTEX Conference, Roubaix, June".

[18] Deng Z.M. and Wang L., (2010). Enhanced visualization of simulated woven fabrics. Fibers \& Polymers, 11, 531-536.

[19] Adabala N., Thalmann N.M., and Fei G., (1995). Realtime Visualization of Woven Textiles. Journal of the Textile Institute, 86, 635-648.

[20] Pascal J., Giralt J., and Brunet P., (2003). An Interactive Package for the Computer-Aided Design of Woven Fabrics. Computers \& Graphics, 10, 359-368.

[21] Moussa A., Dupont D., Steen D., et al. (2010). Structure analysis and surface simulation of woven fabrics using fast Fourier transform techniques, Journal of the Textile Institute, 101, 556-570.

[22] Özdemir H. and Başer G., (2006). Computer Simulation of Woven Fabric Defects Based on Faulty Yarn Photographs, in "Proceedings of the 21st International Symposium on Computer and Information Sciences-ISCIS'06", Istanbul, November 2006, LNCS Vol. 4263, Springer, Berlin.

[23] Ozkaya Y.A., Acar M., and Jackson M.R., (2007). Hair density distribution profile to evaluate yarn hairiness and its application to fabric simulations. Journal of the Textile Institute, 98, 483-490.

[24] Peirce F.T., (1937). The Geometry of Cloth Structure. Journal of the Textile Institute, 28, T45.

[25] Pike M.C., (1965). Remark on algorithm 145 [d1]: adaptive numerical integration by Simpson's rule. Communications of the ACM, 8, 171.

[26] Shang X., Xu S., and Chen Y., (2000). Mathematics Model and Realizing Method of Woven Fabric Structure Computer Simulation. Journal of Donghua University Natural Science.

[27] Sengupta M., (1990). Mine Environmental Engineering. CRC Press, 2, 62.

[28] Chen X., Knox R. T., McKenna D.F., et al. (1996). Automatic Generation of Weaves for the CAM of $2 D$ and $3 D$ Woven Textile Structures. Journal of the Textile Institute, 87, 357358.

[29] Li, Z., Pan, R., Zhang, J., Li, B., Gao, W. and Bao, W., (2016). Measuring the unevenness of yarn apparent diameter from yarn sequence images. Measurement Science and Technology, 27, 015404.

[30 Li Z., Xiong N., Wang J., et al. (2017). An intelligent computer method for automatic mosaic of sequential slub yarn images based on image processing. Textile Research Journal, 004051751773208.

[31] Bouwmans T., (2011). Recent advanced statistical background modeling for foreground detection: a systematic survey. Recent Patents on Computer Science, 4, 147-176.

[32] Basal G. and Oxenham W., (2006). Effect of some parameters on the structure and properties of vortex spun yarn. Textile Research Journal, 76, 492-499.

[33] Brown M. and Lowe D., (2003). Recognising panorama. In: "Proceedings of international conference on computer vision, pp. 1218-1225".

[34] Gonzalez R.C. and Woods R.E., (2006). Digital Image Processing (3rd Edition). Prentice-Hall, Inc. 